\title{
Motion aftereffects with horizontally moving sound sources in the free field
}

\author{
D. WESLEY GRANTHAM \\ The Bill Wilkerson Hearing and Speech Center, Nashville, Tennessee
}

\begin{abstract}
A horizontally moving sound was presented to an observer seated in the center of an anechoic chamber. The sound, either a $500-\mathrm{Hz}$ low-pass noise or a $6300-\mathrm{Hz}$ high-pass noise, repeatedly traversed a semicircular arc in the observer's front hemifield at ear level (distance: $1.5 \mathrm{~m}$ ). At 10-sec intervals this adaptor was interrupted, and a 750-msec moving probe (a $500-\mathrm{Hz}$ low-pass noise) was presented from a horizontal arc $1.6 \mathrm{~m}$ in front of the observer. During a run, the adaptor was presented at a constant velocity $\left(-200^{\circ}\right.$ to $\left.+200^{\circ} / \mathrm{sec}\right)$, while probes with velocities varying from $-10^{\circ}$ to $+10^{\circ} / \mathrm{sec}$ were presented in a random order. Observers judged the direction of motion (left or right) of each probe. As in the case of stimuli presented over headphones (Grantham \& Wightman, 1979), an auditory motion aftereffect (MAE) occurred: subjects responded "left" to probes more often when the adaptor moved right than when it moved left. When the adaptor and probe were spectrally the same, the MAE was greater than when they were from different spectral regions; the magnitude of this difference depended on adaptor speed and was subjectdependent. It is proposed that there are two components underlying the auditory MAE: (1) a generalized bias to respond that probes move in the direction opposite to that of the adaptor, independent of their spectra; and (2) a loss of sensitivity to the velocity of moving sounds after prolonged exposure to moving sounds having the same spectral content.
\end{abstract}

We have reported the existence of an auditory motion aftereffect (MAE), analogous to the well-known waterfall effect in vision (Grantham \& Wightman, 1979). In the previous study, we exposed subjects to sounds that moved repeatedly through the head in one direction (created by dynamically varying the interaural time differences and interaural level differences of pure tones presented over headphones). Following such exposure, subjects tended to respond that subsequently presented probes moved in the opposite direction. This tendency indicates that an auditory MAE exists, although whether the effect is a sensory one or simply a response bias is not yet clear.

The visual MAE is almost certainly a sensory effect. A generally accepted theory to account for this effect postulates the existence of directionally sensitive analyzers in the visual system (Sekuler \& Pantle, 1967). These analyzers become fatigued from prolonged stimulation by a pattern moving in their preferred direction; subsequently, upon presentation of a neutral (stationary) pattern, activity in the analyzers tuned to the opposite direction dominates, resulting in the perception of movement when none is present. The fact that single units that respond selectively to the direction of a moving target have been found in the cat's cortex (Hubel \& Wiesel, 1962)

This research was supported in part by NIH Grant NS18220. The author thanks Lynn $\mathrm{E}$. Luethke for her valuable assistance in collecting and tabulating the data and Sid P. Bacon for helpful comments on an earlier draft of this paper. Portions of this paper were presented at the 113th meeting of the Acoustical Society of America, Indianapolis, May 1987. The author's address is The Bill Wilkerson Hearing and Speech Center, 1114 19th Avenue South, Nashville, TN 37212. and in the rabbit's retina (Barlow \& Hill, 1963) lends indirect support to such a fatiguing hypothesis.

As mentioned in the earlier paper (Grantham \& Wightman, 1979), single units that are directionally sensitive to moving sound sources have also been found. Such units have been found in the cat's inferior colliculus (Altman, 1968), medial geniculate body (Altman, Syka, \& Shmigidina, 1970), cerebellum (Altman, Bechterev, Radionova, Shmigidina, \& Syka, 1976), and cortex (Sovijarvi \& Hyvarinen, 1974). Given the parallels between the visual and auditory physiological data, it seems reasonable to further investigate the auditory MAE to determine whether the fatiguing hypothesis proposed to account for the visual effect might be an appropriate model for the auditory effect as well.

The auditory MAE we measured in 1979 depended on the velocity of the adaptor (MAE magnitude increased as adaptor velocity increased from $30^{\circ}$ to $200^{\circ} / \mathrm{sec}$ ) and on the frequency of the stimulus (the MAE occurred when adaptor and probe were $500-\mathrm{Hz}$ tones, but not when they were $2000-\mathrm{Hz}$ tones). However, even under the best circumstances, the auditory effect was not as phenomenologically compelling as the visual effect. While the visual MAE can persist for $10 \mathrm{sec}$ or more and affects virtually any visual object in the field (e.g., Sekuler \& Ganz, 1963), the auditory MAE seems to be more subtle in nature, requiring the immediate presentation of special probe stimuli for its existence to be noticed.

Part of the reason for the relative weakness of the auditory MAE might be that the presumed auditory motion analyzers were not optimally stimulated in the Grantham and Wightman (1979) study. Simulating motion with pure 
tones presented over headphones might result in a poor representation of the auditory system's response to real environmental sounds in motion. Not only is the spectral content of tones quite restricted compared to that of natural stimuli, but presenting the stimuli through headphones automatically eliminates many cues that are known to be important in human auditory spatial processing (e.g., Searle, Braida, Davis, \& Colburn, 1976).

The present studv represents another attempt to elicit an auditory MAE, but this time employing stimuli more appropriate for any movement-sensitive mechanism that might exist in the auditory system. Specifically, filtered noises were employed as stimuli, and they were presented to subjects via moving loudspeakers in an anechoic chamber. This type of stimulus affords a rich set of localization cues, including changing pinna-induced spectral cues that simply are not available in the case of headphone simulation.

To determine whether the MAE is frequency-specific, we compared its magnitude under two conditions: (1) the adaptor and subsequent probes were spectrally identical, and (2) the adaptor and probes were from different spectral regions.

\section{METHOD}

\section{Subjects}

Four females with clinically normal hearing, 22-35 years of age, served as subjects. Three of the 4 subjects were experienced in psychoacoustic tasks (Subject K.K. was the exception). They were run individually in sessions that typically lasted $1.5 \mathrm{~h}$. Frequent rest intervals were provided.

\section{Procedure}

As in the Grantham and Wightman (1979) study, a probe procedure was employed to measure the magnitude of the motion aftereffect. A sequence of trials consisted of alternating presentations of an adaptor that moved repeatedly through the subject's front hemifield in a semicircular arc, and a probe that was presented from an area in front of the subject. The angular velocity of the adaptor was held constant through the sequence of trials at $-200^{\circ},-150^{\circ}$, $-100^{\circ},-50^{\circ},-35^{\circ},-20^{\circ}, 0^{\circ},+20^{\circ},+35^{\circ},+50^{\circ},+100^{\circ}$, $+150^{\circ}$, or $+200^{\circ} / \mathrm{sec}$, where negative velocity indicates movement toward the left and positive velocity indicates movement toward the right.

As shown in Figure 1, the sequence began with an initial 30-sec exposure to the adaptor. Following adaptor offset, a $750-\mathrm{msec}$ probe was presented in front of the subject, and she had to indicate with a buttonpress whether this probe appeared to move toward the left or toward the right. The actual direction of motion of the probe

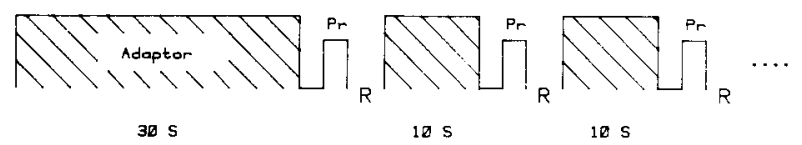

Figure 1. Timing for a sequence of trials in the adaptation paradigm. The hashed areas represent on times for the adaptor; Pr designates the times when a probe is presented (always 750 msec in duration); $R$ indicates the times when a response is expected. The velocity of the adaptor remains constant through a sequence of trials. was determined from trial to trial by random selection of one of five velocities: $-10^{\circ},-5^{\circ}, 0^{\circ},+5^{\circ},+10^{\circ} / \mathrm{sec}$. After the subject's response, the adaptor was turned on again for $10 \mathrm{sec}$, followed by another random-velocity probe and response interval (feedback was not provided). Trials continued in this fashion until four responses had been collected for each of the five probes. After a 2-min rest period, a new adaptor was selected and another sequence of trials was started.

The dependent measure was the percentage of times the subject responded "left," computed for each of the five probe stimuli. The data to be presented are based on 12-20 responses for each probe under each condition of adaptation. It should be noted that, in an unadapted state, the direction of motion of the four moving probes $\left(-10^{\circ},-5^{\circ},+5^{\circ}\right.$, and $\left.+10^{\circ} / \mathrm{sec}\right)$ was correctly identified on $90 \%$ to $100 \%$ of the trials. The subject's responses to the stationary probe $\left(0^{\circ} / \mathrm{sec}\right)$ in an unadapted state give some measure of response bias. ${ }^{1}$

The experiment was conducted in a darkened anechoic chamber, as shown in Figure 2. The subject was seated in the center of the room and instructed to maintain a steady upright, forward orientation through the course of experimentation, without tilting her head either to the side or forward. No bite bar was provided. The adaptor was presented through two loudspeakers (suspended from opposite ends of an overhead boom) that moved around her through a full $360^{\circ}$ in the horizontal plane. The loudspeakers were at ear level and about $1.5 \mathrm{~m}$ from the subject's head. For moving stimuli, only the loudspeaker in the subject's front hemifield was active; when this speaker reached $90^{\circ}$ azimuth, the signal was switched to the opposite loudspeaker that was emerging into the front hemifield on the other side. In this way, the adaptation period consisted of movement in only one direction; the potentially confusing cue from the opposite-direction motion of the speaker behind the subject was absent. ${ }^{2}$

For the special case of a nonmoving $\left(0^{\circ} / \mathrm{sec}\right)$ adaptor, the adaptor was presented from a stationary loudspeaker directly in front of the subject. The opposite loudspeaker (behind the subject) was not used in this case.

The probe was presented through two fixed loudspeakers situated in front of the subject $\left(1.6 \mathrm{~m}\right.$ distant) and separated by $7.5^{\circ}$. These loudspeakers, also at ear level, were 2 of a stationary array of 13 loudspeakers forming a horizontal arc just outside the arc described by the loudspeakers on the boom (see Figure 2). Only the two loudspeakers immediately flanking the center one were employed for probe presentation. Movement for the probe was simulated using stereophonic balancing algorithms (Bauer, 1961); for low-frequency stimuli, fading the left loudspeaker on while fading the right loudspeaker off not only results in a realistic perception of an auditory source moving from right to left, but also yields waveforms at the two ears that are very similar to those that would be produced by actually moving sounds (Grantham, 1986).

\section{Stimuli}

The probe was a $500-\mathrm{Hz}$ low-pass Gaussian noise (designated "Lo"). The adaptor was either the same $500-\mathrm{Hz}$ low-pass noise or a $6300-\mathrm{Hz}$ high-pass Gaussian noise (designated " $\mathrm{Hi}$ ”). Thus, for the low-frequency adaptor condition, the adaptor and probe were spectrally identical (same-spectrum, or Lo-Lo, condition); for the high-frequency adaptor condition, the adaptor and probe were spectrally different (crossed-spectrum, or Hi-Lo, condition) ${ }^{3}$

The signals were presented via 4-in. JBL-8110H high-compliance, full-range loudspeakers, arranged in the anechoic chamber as described above. For both noises, the filter rolloff was $24 \mathrm{~dB} /$ octave, such that there was very little spectral overlap between the two stimuli (Figure 3). Overall level of each sound, presented from a fixed loudspeaker at $0^{\circ}$ azimuth and measured at the subject's head position (1.6 m distant), was $55 \mathrm{dBA}$. 


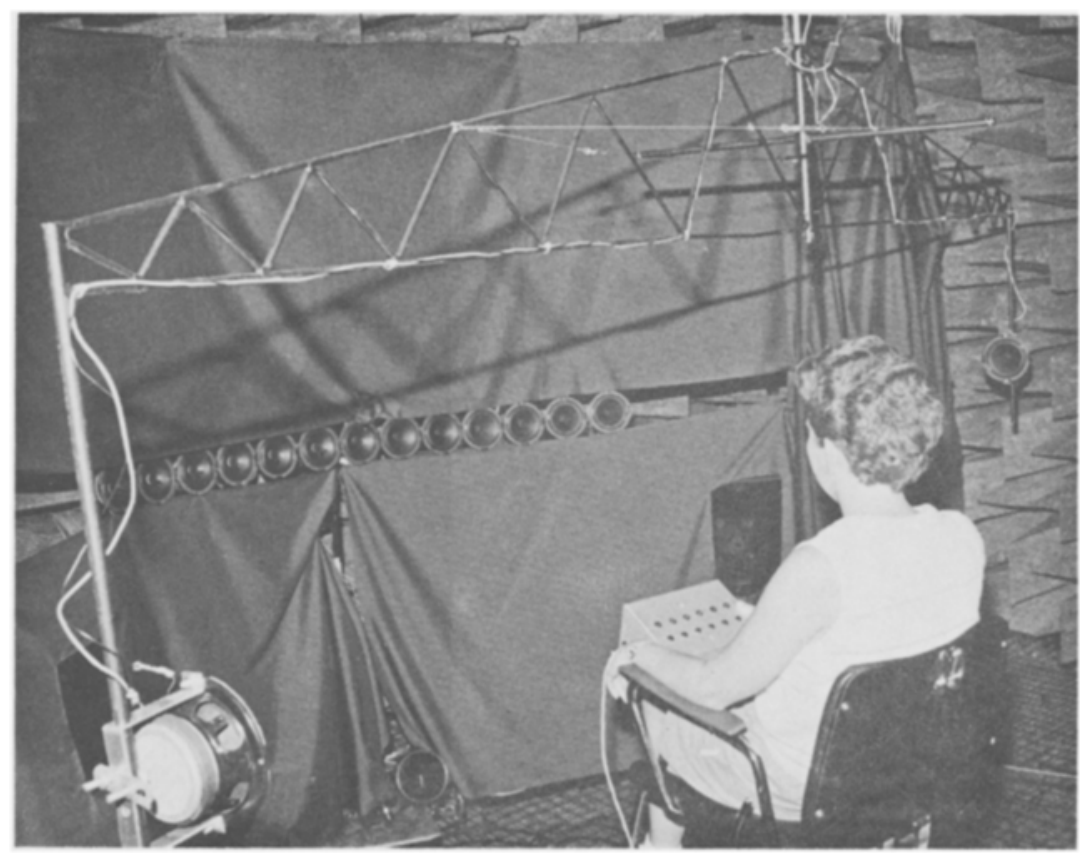

Figure 2. Loudspeaker arrangement in the anechoic chamber. The adaptor is presented through the two loudspeakers suspended from the ends of the overhead boom. This boom moves around a pivot point directly over the subject's head. The probes are presented through two of the fixed loudspeakers forming the array facing the subject.

\section{RESULTS}

Data from Subject K.J. are displayed in Figures 4 and 5 , with percent "Left" responses plotted as a function of probe velocity for the various adaptor velocities. Figure 4 shows the data from the Lo-Lo condition, and Figure 5 shows the data from the Hi-Lo condition. Each panel shows the responses for two adaptors that moved at the same speed, but in opposite directions. In addition, Figure 4 displays, in one panel, performance in the control condition (filled circles: adaptor velocity $=0 \% / \mathrm{sec}$ ), in which the adaptor was presented from a stationary loudspeaker directly in front of the subject.

Looking first at the Lo-Lo condition (Figure 4), a consistent pattern emerges from these data. For adaptor speeds of $35 \% / \mathrm{sec}$ or greater, this subject generally responded "left" more often when adapted to rightmoving adaptors (filled squares) than she did in the control condition (i.e., the functions were displaced upwards on this coordinate system). On the other hand, when adapted to left-moving adaptors (open squares), she generally responded "right" more often than she did in the control condition (the functions were displaced downwards). These displacements reflect the existence of the MAE. To measure the magnitude of the MAE, we can determine the degree to which the filled squares lie above the open squares in the separate panels of Figure 4, or, alternatively, the area between these two functions. For K.J., these areas (expressed as percentages of the total area in the box) were $5 \%, 16 \%, 31 \%, 43 \%, 24 \%$, and
$30 \%$, for adaptor speeds of $25,35,50,100,150$, and $200 \% / \mathrm{sec}$, respectively (Figure 6, first panel, filled symbols). Thus, for this subject there was an initial increase in the magnitude of the aftereffect, followed by a leveling off or slight decrease, as adaptor velocity increased.

In the Hi-Lo condition (Figure 5), using the same measure, the magnitudes of the aftereffects were $8 \%, 12 \%$, $10 \%$, and $4 \%$, for adaptor speeds of $50,100,150$, and $200 \% / \mathrm{sec}$, respectively (Figure 6, first panel, open sym-

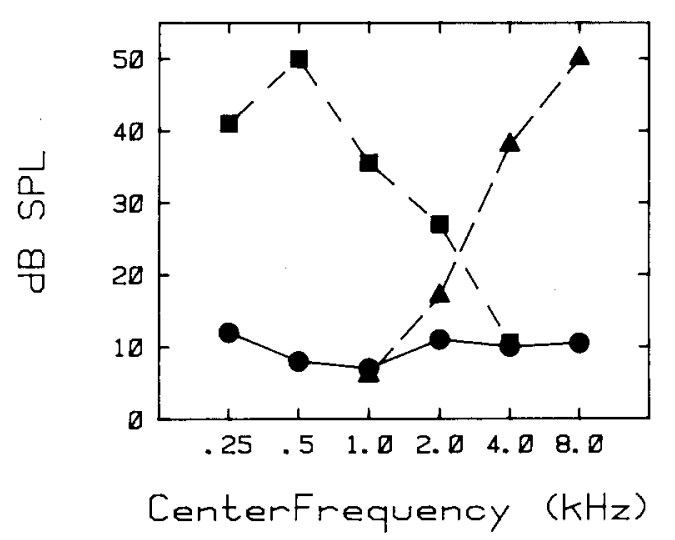

Figure 3. Sound pressure measurements made from the subject's position in the anechoic chamber, using third-octave filter bands. $O=$ in quiet; $\square=500-\mathrm{Hz}$ low-pass noise $(24 \mathrm{~dB} /$ octave attenuation rate) presented from a fixed loudspeaker, $1.6 \mathrm{~m}$ directly in front of the subject; $\Delta=6300-\mathrm{Hz}$ high-pass noise $(24 \mathrm{~dB} /$ octave attenuation rate) presented from the same loudspeaker. 

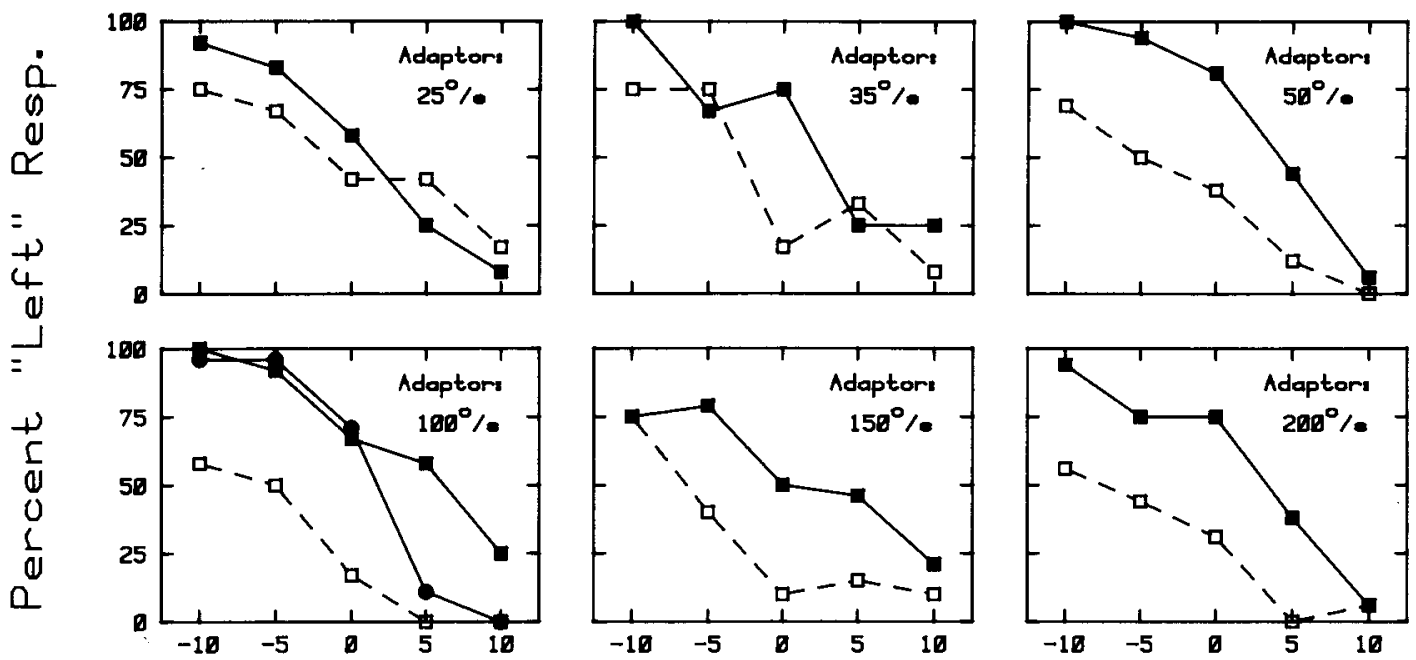

Probe Velocity (deg/s)

Figure 4. Percent "Left" responses as a function of probe velocity for Subject $K$.J. in the Lo-Lo condition (both adaptor and probe were low-pass noises). Each panel displays the results for one adaptor speed. $\square=$ the adaptor moved toward the right at the indicated speed. $\square=$ the adaptor moved toward the left at the indicated speed. $\bullet=$ the adaptor was stationary at $0^{\circ}$ aximuth.

bols). Clearly, the effect was smaller than in the samespectrum condition shown in Figure 4, although it was still consistently positive (the filled squares were above the open squares).

Detailed data from all subjects are presented in Table 1, with percent "Left" responses tabulated for the five probe stimuli for each adaptor velocity and each condition of stimulation (Lo-Lo and Hi-Lo). The general form of the functions from the other 3 subjects was the same as that for K.J. shown in Figures 4 and 5.

As a summary picture of all data, MAE magnitude (the area measure described above) is plotted in Figure 6 as a function of adaptor speed. The four panels display the data separately for the 4 subjects; the parameter is the condition. As can be seen, all subjects showed a consistently larger aftereffect for the same-spectrum condition (Lo-Lo)

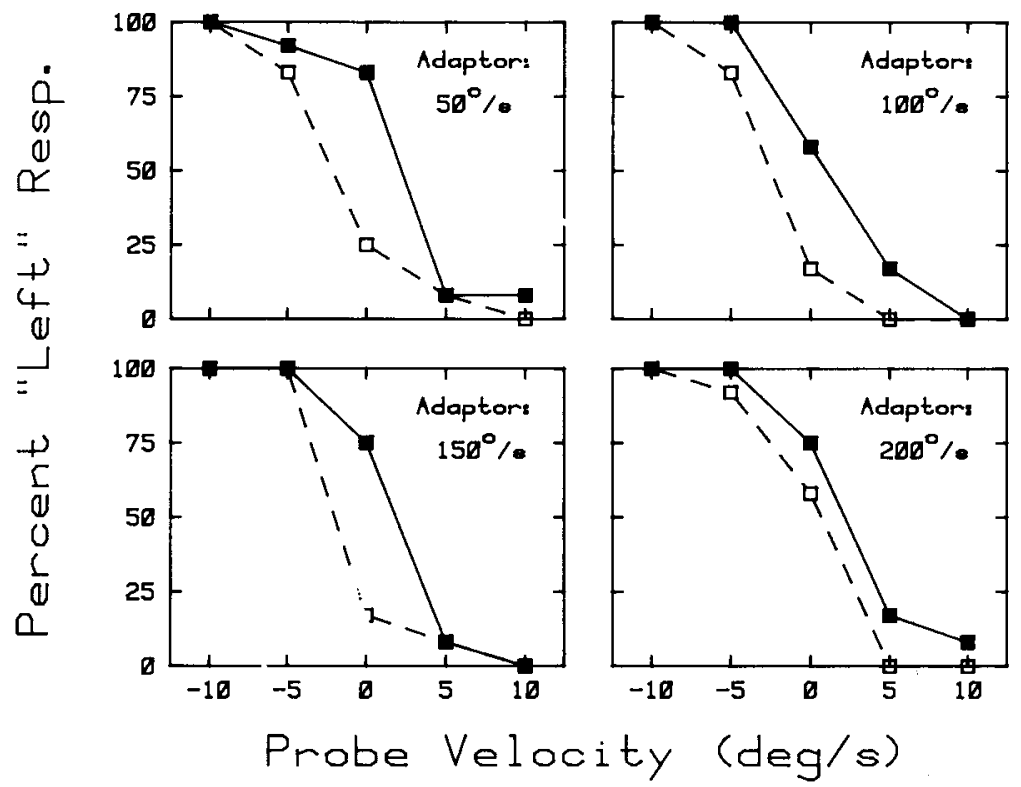

Figure 5. Percent "Left" responses as a function of probe velocity for Subject $K . J$. in the Hi-Lo condition (the adaptor was the high-pass noise and the probe was the low-pass noise). Each panel displays the results for one adaptor speed. $\square=$ the adaptor moved toward the right at the indicated speed. $\square=$ the adaptor moved toward the left at the indicated speed. 


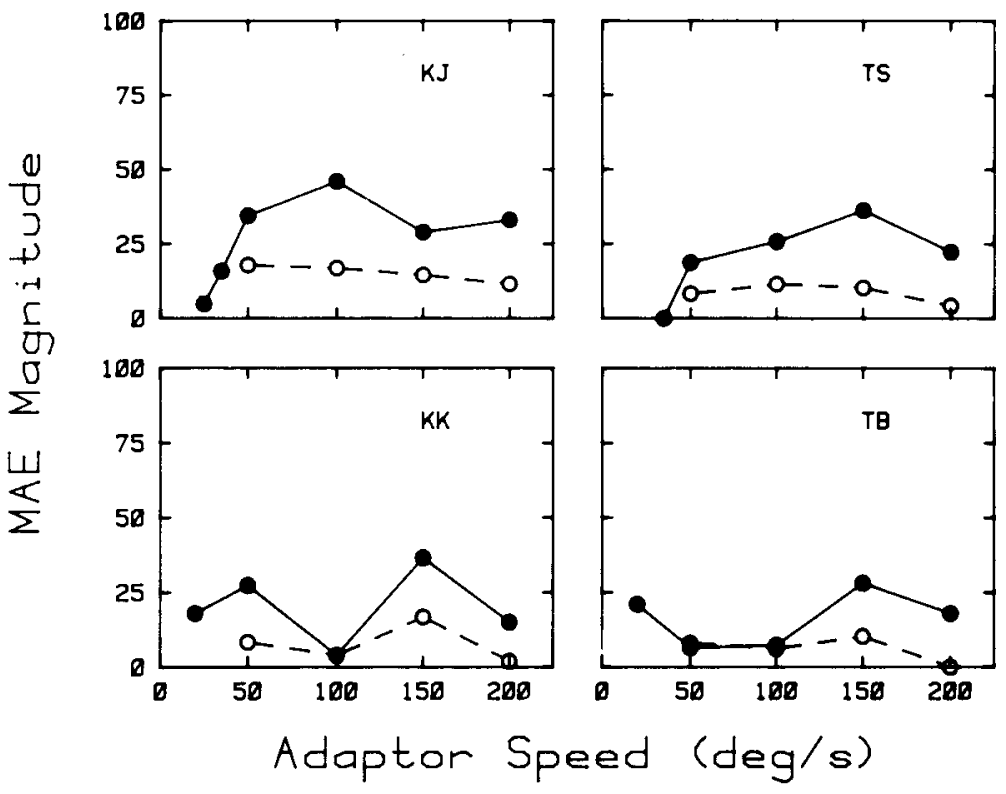

Figure 6. Magnitude of the aftereffect as a function of adaptor speed. Data from the 4 subjects are displayed separately in the four panels. Magnitude is defined bere as the area between the closed squares and open squares in Figures 4 and 5, expressed as a percentage of the total area in the box (a magnitude of 100 would be obtained if all filled squares were at $100 \%$ and all open squares at $0 \%$ ). $O=L_{0}-\mathrm{Lo}$ condition; $\mathrm{O}=\mathrm{Hi}$-Lo condition.

than they did for the crossed-spectrum condition (Hi-Lo), although the pattern of differences was different for each subject.

\section{DISCUSSION}

The form of the functions shown in Figures 4 and 5 and tabulated in Table 1 is the same as that obtained by Grantham and Wightman (1979) in the headphone study: Under control conditions, the subjects responded correctly most of the time to the moving probes, resulting in ogival functions with fairly steep slopes. Under various conditions of adaptation, the ogival shape of the functions was preserved, but the slopes and positions of the functions were affected in consistent ways, as will be discussed below. The fact that the range of probe velocities required to obtain a complete ogive (in an unadapted state) was smaller-by a factor of four-in the present study $\left(-10^{\circ}\right.$ to $\left.+10^{\circ} / \mathrm{sec}\right)$ than that in the earlier study $\left(-40^{\circ}\right.$ to $+40 \% \mathrm{sec}$ ) indicates that the presentation of noise stimuli in the free field does indeed provide more adequate stimulation of auditory motion perception mechanisms than does the presentation of tones over headphones.

The data, as summarized in Figure 6, show that in almost all conditions studied there was a positive aftereffect. For the crossed-spectrum condition (open symbols), this effect was small for all subjects $(0 \%-15 \%)$ and was independent of adaptor speed. However, in the samespectrum condition (filled circles), there were two notable points: (1) the effect was usually greater than in the crossed-spectrum condition, and (2) its magnitude depended on the adaptor speed. Furthermore, there were large individual differences in the manner in which the MAE magnitude depended on adaptor speed. For Subjects K.J. and T.S. (Figure 6, top two panels), there was an initial increase in the magnitude of MAE with increasing adaptor speed, followed by a leveling off or slight decrease. For adaptor speeds below $25^{\circ}-35^{\circ} / \mathrm{sec}$, there was apparently no MAE at all. For Subjects K.K. and T.B. (bottom two panels), on the other hand, there was a definite nonmonotonic form to their functions: the largest MAEs occurred with low $\left(20^{\circ}\right.$ or $\left.50^{\circ} / \mathrm{sec}\right)$ and high $(150 \% / \mathrm{sec})$ adaptor speeds; with intermediate adaptor speeds, there was essentially no MAE. It appears that each subject was susceptible to a different auditory velocity in this adaptation paradigm. These kinds of individual differences are reminiscent of those that have been reported in other binaural tasks (e.g., McFadden, Jeffress, \& Russell, 1973).

\section{Sensory Effect or Response Bias?}

One interpretation of these data is that the component of the effect shown in the crossed-spectrum condition (open symbols in Figure 6) is based on a generalized response bias; that is, subjects tend to respond in a direction opposite to that of the adaptor, more or less independently of the stimulus. However, the additional effect shown in the same-spectrum condition (filled symbols) is frequency-specific, and may reflect a true sensory component of the aftereffect. If these data do reflect the fa- 
Table 1

Percent "Left" Responses to Each Probe Stimulus, for Each Adaptor, for Each Condition

\begin{tabular}{|c|c|c|c|c|c|c|c|c|c|c|}
\hline \multirow{3}{*}{$\begin{array}{l}\text { Adaptor } \\
\text { Velocity } \\
\text { (deg/sec) }\end{array}$} & \multicolumn{10}{|c|}{ Probe Velocity (deg/sec) } \\
\hline & \multicolumn{5}{|c|}{ Lo-Lo } & \multicolumn{5}{|c|}{ Hi-Lo } \\
\hline & -10 & -5 & 0 & +5 & +10 & -10 & -5 & 0 & +5 & +10 \\
\hline \multicolumn{11}{|c|}{ Subject K.J. } \\
\hline-200 & 56 & 44 & 31 & 0 & 6 & 100 & 92 & 58 & 0 & \\
\hline-150 & 75 & 40 & 10 & 15 & 10 & 100 & 100 & 17 & 8 & \\
\hline-100 & 58 & 50 & 17 & 0 & 0 & 100 & 75 & 33 & 0 & \\
\hline-50 & 69 & 50 & 38 & 12 & 0 & 100 & 83 & 25 & 8 & \\
\hline-35 & 75 & 75 & 17 & 33 & 8 & & & & & \\
\hline-25 & 75 & 67 & 42 & 42 & 17 & & & & & \\
\hline 0 & 96 & 96 & 71 & 11 & 0 & 100 & 75 & 42 & 33 & \\
\hline+25 & 92 & 83 & 58 & 25 & 8 & & & & & \\
\hline+35 & 100 & 67 & 75 & 25 & 25 & & & & & \\
\hline+50 & 100 & 94 & 81 & 44 & 6 & 100 & 92 & 83 & 8 & \\
\hline+100 & 100 & 92 & 67 & 58 & 25 & 100 & 100 & 58 & 17 & \\
\hline+150 & 75 & 79 & 50 & 46 & 21 & 100 & 100 & 75 & 8 & \\
\hline+200 & 94 & 75 & 75 & 38 & 6 & 100 & 100 & 75 & 17 & \\
\hline
\end{tabular}

Subject T.S.

$\begin{array}{rrrrrrrrrrr}-200 & 100 & 100 & 42 & 0 & 0 & 100 & 83 & 0 & 0 & 0 \\ -150 & 63 & 56 & 25 & 0 & 0 & 100 & 75 & 17 & 0 & 0 \\ -100 & 88 & 75 & 13 & 6 & 0 & 100 & 100 & 17 & 8 & 8 \\ -50 & 94 & 94 & 38 & 0 & 0 & 100 & 92 & 25 & 0 & 0 \\ -35 & 100 & 88 & 38 & 38 & 6 & & & & & \\ 0 & 100 & 88 & 25 & 0 & 13 & 100 & 100 & 17 & 0 & 0 \\ +35 & 100 & 94 & 56 & 13 & 6 & & & & & \\ +50 & 100 & 94 & 69 & 38 & 6 & 100 & 100 & 42 & 8 & 0 \\ +100 & 100 & 94 & 69 & 25 & 6 & 100 & 100 & 67 & 8 & 0 \\ +150 & 100 & 88 & 88 & 25 & 13 & 100 & 100 & 33 & 0 & 0 \\ +200 & 100 & 100 & 94 & 31 & 12 & 100 & 83 & 17 & 0 & 0\end{array}$

Subject K.K.

$\begin{array}{rrrrrrrrrrr}-200 & 100 & 83 & 25 & 8 & 0 & 100 & 100 & 50 & 0 & 0 \\ -150 & 92 & 58 & 8 & 0 & 0 & 100 & 100 & 8 & 0 & 0 \\ -100 & 100 & 83 & 17 & 0 & 0 & 100 & 100 & 25 & 0 & 0 \\ -50 & 100 & 75 & 17 & 8 & 0 & 100 & 92 & 42 & 8 & 0 \\ -20 & 100 & 83 & 25 & 0 & 0 & & & & & \\ 0 & 100 & 100 & 42 & 0 & 0 & 100 & 92 & 42 & 0 & 0 \\ +20 & 92 & 92 & 75 & 17 & 0 & & & & & \\ +50 & 100 & 100 & 92 & 17 & 0 & 100 & 100 & 58 & 17 & 0 \\ +100 & 100 & 83 & 33 & 0 & 0 & 100 & 100 & 33 & 8 & 0 \\ +150 & 100 & 100 & 75 & 33 & 0 & 100 & 100 & 75 & 0 & 0 \\ +200 & 100 & 92 & 67 & 17 & 0 & 100 & 83 & 75 & 0 & 0\end{array}$

Subject T.B.

\begin{tabular}{rrrrrrrrrrr}
-200 & 92 & 75 & 25 & 8 & 0 & 92 & 83 & 50 & 8 & 0 \\
-150 & 75 & 33 & 17 & 0 & 0 & 100 & 92 & 50 & 0 & 0 \\
-100 & 100 & 75 & 42 & 0 & 0 & 100 & 75 & 42 & 8 & 0 \\
-50 & 92 & 75 & 67 & 0 & 0 & 100 & 92 & 42 & 0 & 0 \\
-20 & 100 & 75 & 33 & 8 & 0 & & & & & \\
0 & 100 & 83 & 58 & 17 & 0 & 100 & 100 & 50 & 0 & 0 \\
+20 & 100 & 92 & 83 & 25 & 0 & & & & & \\
+50 & 92 & 83 & 67 & 18 & 0 & 100 & 100 & 58 & 8 & 0 \\
+100 & 100 & 100 & 42 & 0 & 8 & 100 & 92 & 58 & 0 & 0 \\
+150 & 100 & 83 & 50 & 17 & 0 & 100 & 100 & 75 & 8 & 0 \\
+200 & 83 & 100 & 67 & 17 & 0 & 92 & 75 & 58 & 8 & 0 \\
\hline
\end{tabular}

Note-Each entry is based on 12-20 responses.

tiguing of motion sensitive mechanisms in the auditory system, the form of the functions in Figure 6 suggests that such motion mechanisms are broadly "tuned" with respect to velocity, and that different individuals may in fact be tuned to, or maximally sensitive to, different speeds of motion.
From these data it is difficult to say how such a sensory component underlying the motion aftereffect might operate. It seems not to be the case that exposure to a moving sound source results in a motion aftereffect that simply adds algebraically to real motion. If this were the case, functions such as those shown in Figure 4 would all be 
parallel to the control function; that is, adaptation would result in a horizontal shift of the control function, but would not alter its slope. The fact that most of the adapted functions for all subjects were shallower than their control functions implies that not only was there a tendency to respond in a direction opposite to that of the adaptor, but also that the subjects' discrimination of velocity differences was adversely affected by exposure to movement.

Close inspection of the data indicates that most of the (small) aftereffect measured in the crossed conditions was based on responses to the stationary probe; responses to the four moving probes seemed not to depend so much on the direction of the adaptor (Figure 5). If the crossedspectrum aftereffect is a measure of the generalized response bias produced by exposure to auditory motion, then this bias seems to be revealed primarily in subjects' tendencies to respond that a stationary probe appears to move in a direction opposite to that of the adaptor. The generally greater aftereffect observed in the samespectrum condition can largely be accounted for by the difference in responses to the moving probes (particularly to the $\pm 5 \%$ sec probes) for adaptors moving in opposite directions. In particular, as shown in the lower panels of Figure 4 , a $\pm 5^{\circ} / \mathrm{sec}$ probe whose direction was easily recognized when the subject had not been exposed to motion was responded to at only a chance level when its direction was the same as the adaptor's (the open squares plotted at $-5 \%$ sec and the closed squares plotted at $+5^{\circ} / \mathrm{sec}$ ). Thus, for some speeds, prolonged exposure to a moving adaptor reduced the subject's sensitivity to the motion of a probe whose frequency composition and direction were the same as those of the adaptor.

\section{The Strength of the Auditory Motion Aftereffect}

Although the auditory MAE measured in this study was positive and real, one does not have the same sense of robustness with this MAE that one gets when describing the visual MAE. Part of the difference in the apparent strengths of the visual and auditory MAEs probably rests in the nature of the respective modalities themselves. In both cases, in order to experience the MAE, one needs a test field of stationary objects. In the case of vision, such a test field is a normal situation-most of the time, our visual fields are full of objects that are not only spatially fixed, but also are otherwise temporally invariant. In the case of audition, on the other hand, such a test field is probably not so common. Auditory "objects" are likely to be spectrally dynamic and therefore, perhaps, not able to provide an optimum test situation even if stationary. Although the hum of a fan or other motor would provide the requisite stimulus, the temporal juxtaposition of this kind of test object with a previously occurring, spectrally similar moving auditory event would occur less often in the environment than the visual analogues that first prompted investigation of the MAE more than a century ago. These ideas are merely corollary, of course, to the main fact that the visual modality is specialized for spatial perception, whereas the auditory modality is not. Nevertheless, when provided with the appropriate stimulation in the laboratory, the auditory MAE can be quite evident, as seen in the consistently positive MAEs shown in Figure 6.

Another aspect of the visual MAE that contributes to its description as "robust" is its persistence: In a typical situation, it can last for a minute or more. Although the duration of the auditory MAE was not measured in the present study, it is almost certainly more transitory than the visual MAE, at least with the mode of stimulation employed here. In this study, we have employed a stimulus that is more adequate for the stimulation of auditory motion perception mechanisms that that employed in our earlier study (Grantham \& Wightman, 1979); however, one could design an even better auditory stimulus (employing a circulating array of loudspeakers, for example) that would be more comparable to the usual moving visual pattern employed to evoke the MAE. In this case, it is likely that a stronger auditory MAE would result that might persist for durations analogous to those measured for the visual MAE.

\section{CONCLUSIONS}

The auditory MAE measured in the current paradigm appears to be a combination of at least two effects: (1) a response bias, revealed in the subjects' tendencies to say that a stationary probe moved in a direction opposite to that of the adaptor; and (2) a sensory effect, revealed in subjects' loss of sensitivity to the velocity of auditory motion after prolonged exposure to motion. We suspect that the aftereffect measured under the same-spectrum condition is based on both of these factors, while that measured under the crossed-spectrum conditions is based only on the first factor. The loss of sensitivity appears to occur only when the adaptor and probe have the same spectral content and only for some adaptor velocities. ${ }^{4} \mathrm{~A}$ direct test of this hypothesis can be done by comparing velocity thresholds for horizontally moving sounds (e.g., Grantham, 1986) determined under conditions of (1) no adaptation, (2) adaptation to a moving stimulus that is spectrally identical to that of the probe, and (3) adaptation to a moving stimulus that is from a different spectral region from that of the probe. Under the current hypothesis, velocity thresholds should be elevated only under the second (same-spectrum) condition.

\section{REFERENCES}

Altman, J. A. (1968). Are there neurons detecting direction of sound source motion? Experimental Neurology, 22, 13-25.

Altman, J. A., Bechterev, N. N., Radionova, E. A., Shmigidina, G. N., \& SYKA, J. (1976). Electrical responses of the auditory area of the cerebellar cortex to acoustic stimulation. Experimental Brain Research, 26, 285-298. 
Altman, J. A., Syka, J., \& Shmigidina, G. N. (1970). Neuronal activity in the medial geniculate body of the cat during monaural and binaural stimulation. Experimental Brain Research, 10, 81-93.

BARLow, H. B., \& Hill, R. M. (1963). Evidence for a physiological explanation of the waterfall phenomenon and figural aftereffects. Nature, 200, 1345-1347.

Bauer, B. B. (1961). Phasor analysis of some stereophonic phenomena. Journal of the Acoustical Society of America, 33, 1536-1539.

Ehrenstein, W. H. (1978). Direction-specific acoustical aftereffects. Joumal of the Acoustical Society of America, 64(Suppl. 1), S35.

Grantham, D. W. (1986). Detection and discrimination of simulated motion of auditory targets in the horizontal plane. Journal of the Acoustical Society of America, 79, 1939-1949.

Grantham, D. W., \& Wightman, F. L. (1979). Auditory motion aftereffects. Perception \& Psychophysics, 26, 403-408.

Hubel, D. H., WIESEL, T. N. (1962). Receptive fields, binocular interaction, and functional architecture in the cat's visual cortex. Journal of Physiology, 160, 106-154.

MCFAdDEN, D., JefFress, L. A., Russell, W. E. (1973). Individual differences in sensitivity to interaural differences in time and level. Perceptual \& Motor Skills, 37, 755-761.

Searle, L. D., Braida, L. D., Davis, M. F., \& Colburn, H. S. (1976). Model for auditory localization. Journal of the Acoustical Society of America, 60, 1164-1175.

SEKULER, R. W., \&ANz, L. (1963). Aftereffect of seen motion with a stabilized retinal image. Science, 139, 419-420.

Sekuler, R., \& PANTLE, A. (1967). A model for after-effects of seen movement. Vision Research, 7, 427-439.

Soviuarvi, A. R. A., \& Hrvarnnen, J. (1974). Auditory cortical neurons in the cat sensitive to the direction of sound source movement. Brain Research, 73, 455-471.

\section{NOTES}

1. A single-interval forced-choice ("left" vs. "right") probe procedure was chosen for this experiment, because it had been employed in the Grantham and Wightman (1979) study. This method has the advantage of providing a more objective measure of MAE magnitude (given the relative weakness of the auditory effect investigated under headphones) than does the commonly used measure to quantify the visual MAE, the duration of the aftereffect. Unfortunately, a disadvantage of the single-interval procedure is that it does not allow a separation of sensory capability from response bias in judging motion directionality. A two-interval forced-choice experiment, in which subjects would judge which of two intervals contained a moving target, would provide a biasfree measure of velocity discriminability under conditions of motion adap- tation (such an experiment is currently underway in our laboratory). However, such a two-interval procedure would not address the primary issue of interest here: How does exposure to moving stimuli affect our subsequent judgments of directionality? The single-interval paradigm is the more appropriate one for addressing this question.

2. Ehrenstein (1978) reported that exposure to a sound that moved through a full $360^{\circ}$ around the subject resulted in a localization bias (in the direction opposite to that of the adaptor), but that no motion aftereffect resulted. Part of the reason for his negative finding might have been that the adapting signal remained on while in the rear hemifield. In terms of the changing interaural difference cues, this half of the rotation cycle results in directional information opposite to that provided by the front hemifield sweep. Hence, the alternating left-to-right and right-to-left stimulation might have prevented any motion-sensitive mechanism from becoming adapted. In addition, Ehrenstein apparently employed a localization task that might not have been appropriate for detecting the existence of a motion aftereffect.

3. Some data were also collected with the $6300-\mathrm{Hz}$ high-pass noise as probe, and with either the $500-\mathrm{Hz}$ low-pass or the $6300-\mathrm{Hz}$ highpass noise as adaptor (i.e., $\mathrm{Lo}-\mathrm{Hi}$ and $\mathrm{Hi}-\mathrm{Hi}$ conditions). The subjects had difficulty responding to the high-frequency probes, even under control conditions; for example, the high-frequency probes moving at $\pm 10 \%$ sec sounded to some subjects as if they began to move in one direction, then reversed and went in the opposite direction. These observations are not surprising, since the stereophonic method employed to simulate movement for the probes is truly valid only for frequencies below $1000 \mathrm{~Hz}$; for higher frequencies, the binaural stimulus arriving at the subject's ears during a two-loudspeaker presentation can actually produce conflicting, or "impossible," cues for localization (Grantham, 1986). For this reason, data from the high-frequency probe conditions are not included in the present study.

4. The implicit assumption here is that loss of sensitivity to velocity occurs only if the adaptor and probe stimulate the same spectral region in the auditory system, be that a high-frequency or low-frequency region. An alternative explanation, suggested by the Grantham and Wightman (1979) results, is that it is the spectrum of the adaptor that is critical: Low-frequency adaptors result in loss of velocity sensitivity (for probes of any spectral content), while high-frequency adaptors do not affect velocity sensitivity. To choose between these alternative explanations, one would need to include $\mathrm{Hi}-\mathrm{Hi}$ and $\mathrm{Lo}-\mathrm{Hi}$ conditions in experiments of the kind described here. Unfortunately, we could not obtain valid data in these conditions (see Note 3 above).

(Manuscript received July 2, 1987; revision accepted for publication July 23,1988 .) 